\title{
The first steps toward a global pandemic: Reconstructing the demographic history of parasite host switches in its native range
}

Maeva Techer ( $\square$ maeva.techer@oist.jp )

Okinawa Institute of Science and Technology https://orcid.org/0000-0001-5417-5103

John Roberts

Commonwealth Scientific \& Industrial Research Organisation

Reed Cartwright

Arizona State University

Alexander Mikheyev

Okinawa Institute of Science and Technology

\section{Article}

Keywords: Host switch, population genomics, demographic history, founder size, sympatry

Posted Date: March 9th, 2021

DOI: https://doi.org/10.21203/rs.3.rs-196900/v1

License: (c) (i) This work is licensed under a Creative Commons Attribution 4.0 International License.

Read Full License

Version of Record: A version of this preprint was published at Molecular Ecology on December 9th, 2021.

See the published version at https://doi.org/10.1111/mec.16322. 


\section{Abstract}

Host switching allows parasites to expand their niches. However, successful switching may require suites of adaptations and may decrease performance on the old host. As a result, reductions in gene flow accompany many host switches, driving speciation. Because host switches tend to be rapid, it is difficult to study them in real time and their demographic parameters remain poorly understood. As a result, fundamental factors that control subsequent parasite evolution, such as the size of the switching population or the extent of immigration from the original host, remain largely unknown. To shed light on the host switching process, we explored how host switches occur in independent host shifts by two ectoparasitic honey bee mites (Varroa destructor and V. jacobsoni). Both switched to the western honey bee (Apis mellifera) after it was brought into contact with their ancestral host (Apis cerana), 70 and 12 years ago, respectively. Varroa destructor subsequently caused worldwide collapses of honey bee populations. Using whole-genome sequencing on 63 mites collected in their native ranges from both the ancestral and novel hosts, we were able to reconstruct the known temporal dynamics of the switch. We further found multiple previously undiscovered mitochondrial lineages on the novel host, along with genetic equivalent of tens of individuals that were involved in the initial host switch. Despite being greatly reduced, some gene flow remains between mites adapted to different hosts. Our findings suggest that while reproductive isolation may facilitate fixation of traits beneficial for exploitation of the new host, ongoing genetic exchange may allow genetic amelioration of inbreeding effects.

\section{Background}

Arms races between parasites and their hosts drive evolutionary innovation. Novel parasites can decimate host populations or drive them extinct unless counter-adaptations evolve. Similarly, parasite evolution accompanies the very act of host switching, since it requires adaptations to novel host physiology to persist and to spread. Because parasite adaptations tend to be host-specific, host switches are often associated with host-associated genetic differentiation and eventual speciation. However, only the endpoint of this process is typically observed, as host switches tend to occur rapidly, and the original host is often unknown. As a result, many unanswered questions remain about how parasites acquire new hosts. For instance, if host switches are accompanied by a bottleneck due to reduced gene flow from the ancestral host, how does the parasite have sufficient genetic diversity to adapt? Does gene flow cease completely, or does it continue at a low level, potentially providing additional genetic material for adaptations?

One of the major limiting factors for host switching is the geographic separation between parasites and potential hosts. Globalization has eased these barriers, sometimes giving rise to pandemics (Hatcher, Dick, and Dunn 2012) As a result, host switches are easier to observe and to study in something approaching real-time. One of the most dramatic and economically important switches involved the two ectoparasitic mite species, Varroa destructor and Varroa jacobsoni, which acquired the western honey bee (Apis mellifera) as a new host, 70 and 12 years ago, respectively (D. L. Anderson and Trueman 2000; Roberts, Anderson, and Tay 2015). V. destructor, in particular, spread worldwide, causing extensive honey 
bee population collapses, whereas $V$. jacobsoni has so far remained in Oceania (Traynor et al. 2020; Noël, Le Conte, and Mondet 2020). Both mites were originally found on the sister species, Apis cerana, and came into contact with A. mellifera, which was brought in for purposes of beekeeping (Crane 1968; Chantawannakul et al. 2016). These two host switches occurred in parallel and relatively recently, allowing the reconstruction of how the host switches took place, using genomic tools.

Both switches have been investigated using microsatellite markers and relatively short mitochondrial markers, which revealed that in both species populations on the new host were strongly differentiated and genetically depauperate (Roberts, Anderson, and Tay 2015; Solignac et al. 2005; Warrit, Smith, and Lekprayoon 2006; Navajas et al. 2010; Beaurepaire et al. 2015; Dietemann et al. 2019). While $V$. destructor, in particular, was described as "quasi-clonal" (Solignac et al. 2005), it successfully spread worldwide and has shown a remarkable ability to parasitize genetically diverse $A$. mellifera populations, as well as to evolve resistance to human counter-measures, such as pesticides (González-Cabrera et al. 2016). How does a bottlenecked species achieve such a level of success? Increasing evidence from population genomic analysis of fungal pathogens suggests that the success of many pathogens appears to rely on maintaining some level of adaptive diversity despite the presence of bottlenecks during host switches (Fry 2016; McMullan et al. 2018), but similar work is lacking in animal systems.

To answer this question and to gain broader insight into how host switches happen, we sequenced nuclear and mitochondrial genomes from sympatric populations of the two mites across Asia and Oceania, collected on both novel and introduced hosts. This allowed us far greater power to examine how the host switch took place with much greater precision than was possible previously. We found strikingly parallel dynamics at play in both host switches, which were characterized by a surprisingly large effective population size at the time of the switch and ongoing gene flow with cryptic population genetic processes that may have helped Varroa succeed.

\section{Results}

\section{Cryptic diversity in mitogenomes suggests multiple foundresses at the origins of the host switch}

We examined the genome-wide variation and divergence among $31 \mathrm{~V}$. destructor and $32 \mathrm{~V}$. jacobsoni females from their original and novel hosts (Table 1). Varroa species identity was confirmed by extracting and aligning mitogenomes together with known reference sequences of the mtDNA COX1 458-bp standard marker (Table S1 and Figure S1). A sole mismatch between taxonomic identification and mtDNA barcoding was detected in a $V$. jacobsoni specimen (THV003_1), which was collected in Thailand on $A$. mellifera, though its reproductive status was not confirmed.

Using the set of 2,091 SNPs detected across the mitogenome, we reconstructed the phylogenetic relationship among Varroa lineages for each species (Figure 1). While exhibiting more genomic variability than previously reported, the mitogenome sequences networks were consistent with the standard 
classification of Varroa geographic lineages. We found that both sister species on $3.6 \%$ of mitochondrial consensus sequences. Relative to these levels, divergence within $V$. destructor was very low $(0.5 \%$ with 85 SNPs) but moderate in $V$. jacobsoni with up to $1.3 \%$ (216 SNPs).

We identified 18 distinct mitogenome sequences (thereafter named mitotypes) for $V$. destructor that clustered into seven lineages (Figure 1A and Table S2). Throughout our Asian sampling sites, all $V$. destructor mitotypes on A. mellifera host exclusively belonged to the host switched Korean $\mathrm{K} 1$ lineage, which was also found in one A. cerana sample (Figure 1B). We found three additional derived K1 mitotypes in China that were not previously reported (Table S1). We confirmed that the SNPS differentiating these $\mathrm{K} 1$ mitotypes were not artefacts by manually checking raw reads mapping (cut-off 200x). Interestingly, the two publicly available mitochondrial genomes of the K1 haplotype (France: NC_004454.2 and Japan: AP0195523.1) appear slightly divergent from the dominant K1 mitotype. Though we found the other reported host switch Japanese $\mathrm{J} 1$ lineage in the source population from Japan, we did not detect it on $A$. mellifera. In addition, a re-analysis of 22 COX1 sequences available on $\mathrm{NCBI}$, combined with our new data also indicates diverse mitochondrial backgrounds of host-switched bees, both in $V$. destructor and $V$. jacobsoni (Figure S1).

Considering a similar sample size as for $V$. destructor, we found a much higher genomic variability in the mtDNA in $V$. jacobsoni samples. Our analysis detected 27 unique mitotypes that belonged to seven lineages (Figure 1C and Table S2). Unexpectedly, we found that the misidentified mite on A. mellifera in Thailand (THV003_1) clustered with a new lineage VJ North Thailand 4 (Table 1). This lineage was closely related to the North Thailand 1-3 COX1 (Warrit, Smith, and Lekprayoon 2006; Dietemann et al. 2019) reference sequences (Figure S1) on and Laos 1 mitotypes (Figure $1 C$ ). All other $V$. jacobsoni mites collected on $A$. mellifera $(N=10)$, unequivocally belonged to the reported host switched lineage Papua New Guinea 1. The median joining network showed that six mitotypes found in the A. mellifera population were derived (up to 8 SNPs difference) or identical to native $V$. jacobsoni mitotypes. Two founders PNG 1 mitotypes detected during the host switch event (2008) were retrieved several generations later in A. mellifera colonies (2014) (Table S1).

\section{Host-associated genomic differentiation following host shifts}

Our variant call analysis revealed that 2,728,471 biallelic SNPs in whole-genome sequences of Varroa mites, following a quality and coverage bias filtering. However, multiple results pointed toward a loss a genome-wide loss of variability associated with the transition from the original host $A$. cerana to the novel one $A$. mellifera. When looking at polymorphic sites only, a 2.8 fold reduction in SNPs numbers between was observed in $V$. destructor $\left(N_{V \text { desAcer }}=1,219,990\right.$ vs $N_{V \text { desAmel }}=429,420$ SNPs $)$ and 3.6 fold in $V$. jacobsoni $\left(\mathrm{N}_{\mathrm{Vdes} A c e r}=1,546,366\right.$ vs $\left.\mathrm{N}_{\mathrm{Vdes} A m e l}=630,534 \mathrm{SNPs}\right)$. Reduction of genetic diversity with the founding of new populations was also observed with the average genomic nucleotide diversity $\pi$ in both $V$. destructor $\left(\pi_{\mathrm{V} \text { desAcer }}=0.0020 \mathrm{vs} \pi_{\mathrm{Vdes}}\right.$ Amel $\left.=0.0005\right)$ and $V$. jacobsoni $\left(\pi_{\mathrm{VjacAcer}}=0.0014 \mathrm{vs}\right.$ 
$\left.\pi_{V j a c A m e l}=0.0008\right)$. Genome scans using $20 \mathrm{~kb}$ sliding windows showed that $\pi$ values in the new host was overall lower than in the original host but that some regions exhibited higher nucleotide diversity (Figure S2). Analysis of the genomic heterozygosity revealed extremely high levels of inbreeding coefficient in mite genomes with the lowest value $\mathrm{F}=0.686$ in $V$. destructor and $\mathrm{F}=0.721$ in $V$. jacobsoni (Table S3). However, when comparing A. mellifera and $A$. cerana host populations, we found no significant difference in $\mathrm{F}$ estimates for $V$. destructor (two-tailed t-test $p$-value $=0.263$ ) and $V$. jacobsoni (two-tailed t-test $p$-value $=0.297$ ). Such levels in both species were previously reported using microsatellites (Roberts, Anderson, and Tay 2015) and more likely resulting from incestuous Varroa mating.

PCA further illustrated the loss of genetic diversity in host-switched mites coupled with genome-wide differentiation (Figure 2AC). $F_{S T}$ and $D_{x y}$ pairwise estimates indicated strong differentiation between nuclear genomes from divergent lineages (Table S4). Within each species, high levels of genetic differentiation all along all seven chromosomes were detected between host-adapted lineages (Figure S2). Interestingly, when comparing the host-switched $V$. destructor specimens to native-host samples from the same mitotype $(\mathrm{K} 1)$ there was far less genetic differentiation $\left(F_{S T}=0.004\right)$ compared with any other native-range population $\left(F_{S T}>0.6\right)$ (Table S4). Thus, host-switched populations still carry many of the genes present in the population of origin, a scenario consistent with rapid reproductive isolation postswitch. Similar patterns were obtained genome-wide for the larger sliding windows of $20 \mathrm{~kb}$ of $\mathrm{F}_{\mathrm{ST}}$ and $\mathrm{D}_{\mathrm{xy}}$ estimates show that $A$. cerana and $A$. mellifera $\mathrm{K} 1$ mites diverged massively (Supplemental online markdown).

We found similar patterns of population structure and divergence among hosts and mtDNA lineages in $V$. jacobsoni, while host switch was more recent. In our study, we did not find clonal genomes in either $V$. jacobsoni or $V$. destructor mites collected from A. mellifera populations.

\section{Ancestry of host switched populations and evidence of gene flow}

NGSadmix analysis conducted on 1,276,602 unlinked biallelic SNPs confirmed the population structure observed between $A$. mellifera and $A$. cerana mites and helped to identify the source of host populations. At $\mathrm{K}=2$, population structure quickly emerged among $V$. destructor mites. $\mathrm{J} 1$ and $\mathrm{K} 1 \mathrm{~A}$. cerana mites were assigned unequivocally to the same genetic cluster as all $\mathrm{K} 1 \mathrm{~A}$. mellifera mites (Figure 2B). Therefore, genome-wide sequencing confirmed the contribution of $\mathrm{K} 1$ and related $\mathrm{J} 1$ mite sources in the founding of novel host populations. In $V$. jacobsoni, admixture estimates suggested that two large genetic clusters exist: Northern/Asian $V$. jacobsoni lineages differing from the South/Oceanian (Figure 2D). At $\mathrm{K}=3$, unreported spillover events were evidenced with the mite (THV003_1) found in A. mellifera in Thailand that was genetically related to mites in Viet Nam (Laos 1). Here, population genomics also supports the origin of $V$. jacobsoni host switch in Papua New Guinea to be exclusively from A. cerana 
PNG1 and Java 1. Unlike in $V$. destructor, signal of recent migration was exposed by one individual (PGV956_4) in A. mellifera Papua New Guinea population.

Recent migration and spillover events were confirmed by analyzing mite's body metagenomic reads. Our predictions that honey bee hosts mtDNA reads could be retrieved from the whole crushed parasitic mite was validated. The proof of concept for this method was first tested and demonstrated with mite families (used for de novo mutation measurements) that were actively feeding at the time of collection (Table S5). For population genomics mites, up to $0.18 \%$ of all reads from each mite library ( $n=115,581$ reads) matched honey bee host mitochondrial reference genomes. Interestingly, the NGSadmix $V$. jacobsoni outlier (PGV956_4) was actually "caught" in migration with more $A$. cerana reads $(N=1,622)$ than of the collected host $A$. mellifera $(N=69)$. While admixture estimates did not spot such outliers in $V$. destructor, the diet molecular analysis revealed reverse cases in Viet Nam (VNV475_1) and in Japan (JPV025_1). A. mellifera and $A$. cerana honey bees present enough important morphological and behavior differences that experts field collectors would have assuredly not missed. Such convergent observations more likely indicated that these mites migrated between hosts shortly before collection.

\section{Low de novo mutation rate from pedigree-based measurements}

We examined whether the rapid parallel differentiation observed between Varroa host populations was driven by high frequency of new mutations by estimating the de novo mutation rate, following a previously published approach (Ramu et al. 2013). As the Varroa reproductive sequence cycle is well known (one haploid son, followed by up to four diploid daughters), we sequenced six diploid mothers and their respective sons at a coverage between $18 x-33 x$. Before filtering, the number of detected mutations was 3,082 but there was extreme variation between samples in the number of mutations called. Notably mother-son pairs with less coverage contained several folds more mutation calls than mother-son pairs with higher coverage. Samples with higher mutation calls also showed stronger evidence of clustering of mutation calls, which typically indicates false-positive mutation calls. After filtering, two mutation calls remained, but showed low coverage and were discarded as unreliable.

We conducted a set of simulations to deal with coverage bias issues in the data set. Out of 6,000 simulated mutations, 3,464 could be detected by our pipeline, indicating that our recall rate was approximately $58 \%$. The total haploid length of the 7 chromosomes considered in the mutation scan was $362,833,908$ base pairs. Given that we found no mutations among 6 mother-son pairs, we estimate that the mutation rate in Varroa is less than $8 \times 10^{-10}$ per bp per generation $\left(\frac{1}{7 \times 362833908 \times 0.5773}\right)$ or less than 0.28 mutations per son per generation.

\section{Estimating of host switch demographic history and founding population size}


We aimed to further parameterize the demography of host switches detected in mitochondrial data using coalescent-based modeling. Demographic inference using fastsimcoal2 was conducted using an isolation with migration scenario (Figure S3). For $V$. destructor, 32 haploid mite genomes were sequenced from $A$. mellifera host and 22 haploid mite genomes were sequenced from $A$. cerana host. For $V$. jacobsoni, 18 haploid genomes of $A$. mellifera mites and 6 haploid genomes of $A$. cerana mites from Papua New Guineas were projected (excluding the older sample from 1997). Given that we were only able to estimate the upper limit of de novo mutation rate, we explored whether lower mutation rate improve the observed maximum likelihood distribution of 100 replicates (Figure S4). We examined rates as low as 1.0 $\times 10^{-11}$, at the lower limit of rates in arthropods (Fazalova and Nevado 2020). We also tested whether including an inbreeding coefficient would help improving the model likelihood by implement $70 \%$ of inbreeding (minimum detected in both species). However, lower mutation rates did not improve model fit for both $V$. destructor (Kruskal-Wallis chi-squared $=497.61, \mathrm{df}=494, p$-value $=0.446$ ) and $V$. jacobsoni (Kruskal-Wallis chi-squared $=499, \mathrm{df}=498, p$-value $=0.479)($ Figure S4). The inbreeding coefficient did also not significantly improve the likelihood with equal mutation rate (available in online supplemental markdown). In addition, 2D-joint SFS visual inspection did not reveal striking differences, and the parameter estimates overall were qualitatively similar over the range of mutation and inbreeding parameters we examined.

Hence, demographic parameters and their bootstraps values were estimated using $\mu=8 \times 10^{-10}$ per bp per generation and no inbreeding value. Mean values and $95 \%$ confidence intervals for each species are graphically summarized in Figure 3. The older host switch with $V$. destructor was estimated to have occurred 887 generations before sampling ( 88 years ago considering 10 generations / year (Cornuet et al. 2006)) with a founder effect lasting for 177 generations. Estimates suggested that 156 [Cl95 = 146165] haploid genomes of $V$. destructor contributed to the founding event in Asia. From that point on, the novel mite population quickly growing on $A$. mellifera diverged from the sympatric $A$. cerana source populations. While the best scenario assumed migration, our results suggest gene flow with low but continuous migrating rates between hosts. Despite being a more recent event, estimated around 183 generations ago ( 18 years ago), demographic parameters for $V$. jacobsoni also supported a large founding size reaching 138 haploid mites. The effective population size of $V$. jacobsoni mites on $A$. mellifera was lower than the source population, contrary to the demographic history estimated for $V$. destructor. Finally, bidirectional migration rates suggested more important ongoing gene flow among host populations.

\section{Discussion}

Virtually every living species has at least one species of parasite, making parasitism perhaps the most successful mode of life. Most studies examine parasitism from either a macroevolutionary perspective, for instance, through host-parasite co-phylogenies, or at the population level, by examining patterns of differentiation between parasites and hosts (Poulin et al. 2011; Penczykowski, Laine, and Koskella 2016). Theoretical modelling links the two scales, suggesting that specialization coupled with trade-offs in 
performance on alternative hosts should lead to speciation (Sexton et al. 2017). Yet, empirical observations of this process remain scarce, particularly of host switch demography, which includes key parameters such as population sizes during switches that are necessary to ensure sufficient evolutionary potential for parasites (McDonald and Linde 2002). For this reason, pandemic spread of Varroa mites among honey bees has long been puzzling, given their "quasi-clonal" structure in the invasive portion of their range (Solignac et al. 2005). Using high-resolution mitochondrial and nuclear genomic data from sympatric populations, we show that in both $V$. destructor and $V$. jacobsoni, (a) genetic bottlenecks were far less severe than previously estimated and (b) while gene flow was greatly reduced post-host switch, consistent with models of incipient speciation that may accompany acquisition of novel hosts, gene flow has not completely stopped. Our data highlight the importance of genetic diversity during initial stages of parasitic host switches.

While loss of genetic diversity is common in parasites, it often occurs in the parasite's introduced geographic range (Auger-Rozenberg et al. 2012; Demastes et al. 2019). Consequently, parasites may possess sufficient genetic diversity to parasitize their hosts. For example, the fungal parasite responsible for ash dieback in Europe arrived from Asia, and while bottlenecked to only two haplotypes, it nonetheless maintains adaptive diversity in key host interaction genes (Fry 2016). To the best of our knowledge, our study is unique in quantifying demographics within the native range of the parasite, where it has opportunities for additional gene flow from genetically diverse sympatric populations. At least for Varroa, the initial host switch requires an unexpected amount of genetic diversity at the point of the switch, but rapidly leads to reproductive isolation between sympatric populations on novel and original hosts. The amount of differentiation appears to increase over time, being greatest in $V$. destructor, which switched $\sim 50$ years earlier than $V$. jacobsoni. Varroa mites coevolve quickly with their new hosts (Beaurepaire et al. 2019) and such sympatric isolation could ultimately lead to speciation.

Post host-switch speciation can occur in the presence of gene flow, if specialization is adaptively favored and selection acts on many genomic regions (Pinho and Hey 2010; Feder, Egan, and Nosil 2012). By promoting genomic heterogeneity and introducing beneficial mutations (Tomasini and Peischl 2020), gene flow can ameliorate lost diversity due to selection or inbreeding (Lenormand 2002; Whiteley et al. 2015), both of which would impact host-switched Varroa populations. Alternatively, de novo mutation rates could contribute to population genetic variation, but our direct estimation of mutation rate did not show exceptionally high mutation rates ( $<\times 10^{-10}$ per bp per generation), compared to other arthropods (H. Liu et al. 2017; Fazalova and Nevado 2020). Consequently, immigration may be the primary means of introducing new alleles into populations on the novel host during early stages of the host switch.

While host switch events by specialized parasites were once assumed to be rare, this assumption is increasingly challenged (Rigaud, Perrot-Minnot, and Brown 2010; Nylin et al. 2018). Ecological fitting theory suggests that shifts could readily occur in species with a pre-existing ability to use novel hosts (Araujo et al. 2015). Host switches in Varroa appear to fit this model. While the western honey bee has been introduced throughout the Varroa native range, mitochondrial data, which are geographically informative (Figure 1), suggest that switches occurred only in Korea, Japan, Philippines (V. destructor), 
Papua New Guinea and possibly Thailand (V. jacobsoni) (Roberts, Anderson, and Tay 2015; Navajas et al. 2010; Beaurepaire et al. 2015; Dietemann et al. 2019). A. cerana subspecies are strongly differentiated geographically (Radloff et al. 2010; K. Tan et al. 2016; Chen et al. 2018; Yancan et al. 2019; llyasov et al. 2019; Ji et al. 2020), and Varroa mitotypes mirror host biogeography and subspecies distribution (Warrit, Smith, and Lekprayoon 2006; Beaurepaire et al. 2015; Rueppell et al. 2011; Dietemann et al. 2019). This suggests that Varroa populations may vary in traits, such as host specificity, as a result of their previous coevolutionary interaction with local $A$. cerana subspecies. This may have allowed some populations to switch, while others were unable, in spite of available novel hosts (W. Li et al. 2019). However, the dynamic nature of the interaction does not preclude additional switches in the future.

One potential limitation of our data set is its sparse geographic and temporal sampling, given the size of the native range. While small sample size is enough to retrace ancestral event, large sample sizes can increase the confidence in model selection and parameter estimates for recent demographic events (Robinson et al. 2014). Yet, past scenario and demographic estimates were consistent with mite biology and reflected direct observations made during their invasion of $A$. mellifera. Furthermore, while our data set captured most of the described Varroa mitochondrial lineages and even discovered new ones (D. L. Anderson and Trueman 2000; Solignac et al. 2005; Dietemann et al. 2019; Zhou et al. 2004), some reported mitotypes were missing. In the future, incorporating larger sample size from modern source populations could help better estimate each lineage genetic contribution to host switch success. In addition it would allow to estimate how host switch may have affected native diversity as for the case of J1 lineage displacement (de Guzman, Rinderer, and Anthony Stelzer 1999; Ogihara et al. 2020). Despite these limitations, our analysis correctly reconstructed known aspects of Varroa host switch demography, such as the times of the switches. However, these times need to be regarded as approximate, since generation times may fluctuate due to a variety of factors, such as seasonality or brood availability. We relied on a previous approximation of 10 generations per year (Cornuet et al. 2006). As a result, we caution against over-relying on the apparent precision of the numerical estimates (Table 2). Nonetheless, data from both mitogenome sequencing and coalescent analysis all point to a relatively large founding population size of the Varroa mites (Figures 1 and 3).

Our findings highlight the dynamic and ongoing nature of host switches in the native range, and the need to better understand native mite populations. Mitochondrial DNA has already detected the likely presence of several host switches, such as those in Philippines in 2015 (Beaurepaire et al. 2015) and the presence of new haplogroups in Eastern Europe (Gajic et al. 2013), indicating that populations of Varroa are continuously testing $A$. mellifera as a new host, and may have spread without our awareness.

Additionally, while this work provides insight into the initial host switch, the demographics of the subsequent worldwide pandemic remain largely unknown, for instance the critical population size necessary to establish a regional infestation. Future research on global demographic parameters such as genetic diversity and gene flow is crucial (Evans and Cook 2018). Since V. jacobsoni presents striking parallels to $V$. destructor, this knowledge could be applied to forecast and prevent its spread. 


\section{Materials And Methods}

\section{Mite sampling on original and novel honey bee hosts}

We sequenced adult females throughout the Varroa native ranges where original and novel hosts occur in sympatry $(V$. destructor $N=31$ and $V$. jacobsoni $N=32$; Table 1 ). For $V$. destructor, specimens were collected between 1996 and 2003 as part of the taxonomic revision and diversity survey carried by Anderson and Trueman (D. L. Anderson and Trueman 2000) and Navajas et al. (Navajas et al. 2010) and included 14 samples from $A$. cerana and 17 from A. mellifera collected from Japan, South Korea, China, Thailand, Viet Nam, Myanmar and Nepal. For V. jacobsoni, female specimens were obtained from Anderson and Trueman (D. L. Anderson and Trueman 2000) (1994-2004) and Roberts et al. (Roberts, Anderson, and Tay 2015) (2008-2015) surveys of host shifts reports in Papua New Guinea. A total of 19 adult female $V$. jacobsoni mites were collected from $A$. cerana across different areas in India, Thailand, Viet Nam, Malaysia, Indonesia and Papua New Guinea. In contrast to V. destructor, V. jacobsoni infestation on $A$. mellifera is still restricted to Papua New Guinea from which 10 samples were collected. Additionally, we included three $V$. jacobsoni mites collected from an alternative original host $A$. nigrocincta found in Indonesia. A. nigrocincta is not believed to be the source of the host-switched mites, so these samples were included for comparison purposes.

Sampling complete details regarding host, location and year are available in Table S1. Exact geographical coordinates were not always available for samples collected before 2008 , and were approximated by the locality provided, or published survey maps (D. L. Anderson and Trueman 2000; Navajas et al. 2010; Zhou et al. 2004; Denis L. Anderson and Fuchs 1998). All individuals were mature sclerotized females collected from single colonies and were preserved in individual Eppendorf tubes. Each collection tube was stored with $70 \%$ ethanol and kept at $-20^{\circ} \mathrm{C}$ at the CSIRO in Canberra, Australia.

\section{DNA extraction and whole genome resequencing}

Mites were surface sterilized by cleaning them in absolute ethanol using a sterile brush to remove any external debris, and then gently shaken in a $2.0 \mathrm{~mL}$ Eppendorf filled with absolute ethanol. Each mite was then dried for $10 \mathrm{sec}$ on a sterile paper towel before being placed in a $1.5 \mathrm{~mL}$ Eppendorf tube in liquid nitrogen. Genomic DNA was extracted from each mite by crushing the whole body, using a sterile pestle to obtain a fine powder and processed with a QIAamp DNA Micro Kit (๑ Qiagen) following the manufacturer's instructions. Final elution volume was $15 \mu \mathrm{L}$. Total dsDNA was measured using a Qubit ${ }^{\text {tM }}$ 4 Fluorometer with an Invitrogen dsDNA HS Assay Kit.

For population genomic samples, short-inserts of 150-bp paired-end libraries were prepared for each individual using a Nextera XT DNA Library Preparation Kit (Illumina ${ }^{\circledR}$ ). Size-selection and cleanup were accomplished using CA-magnetic beads (Dynabeads ${ }^{\circledR}$ MyOne Carboxylic Acid, Invitrogen), and 11-11.5\% PEG 6000 (Sigma-Aldrich @ LLC). Library quality and size were assessed using a Bioanalyzer High Sensitivity DNA kit (Agilent). Libraries were run on HiSeq 4000 and NovaSeq6000 in 150 bp x 2 paired- 
end mode (Illumina ${ }^{\circledR}$ ) at the OIST Sequencing Center. Biosamples and DRA accession as well as sequencing coverage for each sample are provided in Table S1.

\section{Host DNA contents in mite whole-body metagenomics}

We cross-checked honey bee host identities reported during sampling with host read identity to detect potentially migrating mites. Mites feed on honey bees during the phoretic phase (Ramsey et al. 2019) and maintain a consistent feeding regimen by consuming $\sim 1 \mu \mathrm{L}$ of host fluid (digested fat-body and haemolymph) per day (Posada-Florez et al. 2019). Therefore, we assumed that host DNA would be retrieved from crushed whole-mite tissues. Mitochondrial DNA was targeted, as it is more abundant than nuclear DNA. We mapped raw fastq reads on honey bee host mitochondrial reference genomes using [NC_001566.1] for A. mellifera ligustica (Crozier and Crozier 1993) and [NC_014295] for A. cerana (H.-W. Tan et al. 2011). The number of reads mapped to either one of these honey bee host reference genomes was counted and compared to sampled host identities.

\section{Data filtering, mapping and genotype calling}

Commands used for each analysis step are available on our Snakemake script (Köster and Rahmann 2012) available on https://github.com/MaevaTecher/varroa-host-jump. Briefly, we assessed demultiplexed fastq read quality using FastQC (Andrews and Others 2010). We then mapped reads to the $V$. destructor reference genome on NCBI [GCF_002443255.1] (Techer et al. 2019) separately from the complete mitogenome [NC_004454.2] (Navajas et al. 2002) using the soft-clipping and very sensitive mode of NextGenMap v0.5.0 (Sedlazeck, Rescheneder, and von Haeseler 2013) (following a comparison with Bowtie2 v2.6 (Langmead and Salzberg 2012)). Reads were sorted and duplicates were removed using SAMtools (H. Li et al. 2009), and subsampled to a maximum coverage of 200 using VariantBam (Wala et al. 2016) to speed up processing. Mapping rates and reads depths were computed from the generated BAM files.

We generated three data sets for the mapped reads depending on analysis requirements, following Yamasaki et al. (Yamasaki et al. 2020). First, we obtained a "SNP-only dataset" containing only variant sites using FreeBayes v1.1.0 (Garrison and Marth 2012) with the following parameters: minimum mapping $=10$, minimum base quality $=5$, use of the four best SNP alleles, and no populations prior. In order to correct for coverage bias and sequencing artefacts in problematic regions, we estimated twice the mean reads depth along the seven main chromosomes. Subsequently, variants were filtered using using VCFtools v0.1.12b (Danecek et al. 2011). The "SNP-only dataset" resulted in 2,728,471 SNPs. Second, we computed an "all-sites dataset" by calling both variants and invariants sites using BCFtools v1.9 mpileup (H. Li 2011). We removed indels and sites at over twice the computed mean depth, as well as sites with any missing data. We excluded sites that were not placed on the seven chromosomes. After filtering, the "all-sites dataset" resulted in 2,130,335 SNPs and 120,279,163 monomorphic sites. Third, we obtained a "mtDNA SNP-only dataset" by calling polymorphic sites only on 
the mitochondrial genome NC_004454.2. We used FreeBayes v1.1.0 with strict quality parameters. The dataset resulted in 2,091 SNPs. These were validated in the COX1 region by Sanger sequencing (see next section).

\section{Comparative mitogenomes analysis and lineages identification}

We used the "mtDNA SNP-only dataset" for mitochondrial variation genomic analysis by converting and generating individual nucleotide sequences using the option vcf2fasta in vcflib scripts (github.com/vcflib). Nucleotide sequences were screened and aligned (16,476 bp) using Geneious Prime ${ }^{\circledR}$ 2019.2.3 together with the reference mitochondrial sequences NC_004454.2 (V. destructor on $A$. mellifera, France (Navajas et al. 2002)) and also, to avoid problems with reference bias, from AP019523.1 (V. destructor on A. mellifera, Japan (Harada et al. 2020)). Mitotype diversity for each Varroa species and distribution in hosts was assessed using the median joining network method from SplitsTree5 (Huson and Bryant 2006). Divergence levels between Varroa species was estimated by comparing the wholemitochondrial consensus sequences with Geneious Prime ${ }^{\circledR}$.

Standard methods exists to determine Varroa species and lineage (also named haplogroup) identity by using the following mitochondrial markers: 1) a 458-bp fragment from the COX1 gene (D. L. Anderson and Trueman 2000; Dietemann et al. 2013) and 2) a concatenated 2,696-bp fragment including COX1, ATP6, COX3, and CYTB (Navajas et al. 2010). Thus, we determined the lineage (e.g. Korean K1, Japan J1, etc.) of each mitotype by extracting the COX1 barcoding region of interest and aligning it together with 60 unique COX1 reference sequences from NCBI using ClustalW and manual check. A neighbor-joining tree was computed on the fasta alignment using IQ-tree (Nguyen et al. 2015) and exported with iTOL (Letunic and Bork 2019). Following Varroa revised nomenclature (Traynor et al. 2020), a sequence was considered from a known lineage only if $100 \%$ identical to reference COX 1 haplotype. The same process was used for sub-lineages (e.g. Korean K1-1/K1-2, Korean K1-3,...) identification by with the COX1-ATP6-COX3-CYTB concatenated region sequences which were aligned with 22 available reference concatenated sequences for V. destructor only (Navajas et al. 2010; Gajic et al. 2013; Elbeaino et al. 2016).

To ensure that filtering of the " $m$ tDNA SNP-only dataset" did not remove existing and known variants, we also sequenced the COX1 gene using Sanger sequencing using primers $10 \mathrm{kbCOIF} 1$ and $6,5 \mathrm{KbCOIR}$ (Navajas et al. 2010). PCR reactions were carried out in $25 \mu \mathrm{L}$ containing $5 \mu \mathrm{L}$ of $5 X$ Phusion $\circledast \mathrm{HF}$ buffer; $0.5 \mu \mathrm{L}$ of dNTP mix (10mM); $0.25 \mu \mathrm{L}$ of Phusion ${ }^{\circledR}$ High-Fidelity DNA Polymerase (NEB); $1.25 \mu \mathrm{L}$ of each oligo primer $10 \mathrm{kbCOIF} 1$ and $6,5 \mathrm{KbCOIR}(10 \mathrm{mM}) ; 1 \mu \mathrm{L}$ of template DNA $(0.5 \mathrm{ng} / \mu \mathrm{L})$ and Milli-Q water. Samples were denatured at $98^{\circ} \mathrm{C}$ for $30 \mathrm{sec}$, and then PCR was performed for $35 \mathrm{cycles}$ of $10 \mathrm{sec}$ denaturation at $98^{\circ} \mathrm{C}, 15 \mathrm{sec}$ of annealing at $59^{\circ} \mathrm{C}$ and $15 \mathrm{sec}$ of extension at $72^{\circ} \mathrm{C}$ with a 5 min final elongation at $72^{\circ} \mathrm{C}$. DNA amplification success was visualized by loading $3 \mu \mathrm{L}$ of PCR product with $3 \mu \mathrm{L}$ of loading dye on $1 \%$ agarose gel (110V for $20 \mathrm{~min}$ ). PCR products were then cleaned-up using Dynabeads ${ }^{\circledR}$ MyOne Carboxylic Acid, CA-beads (Invitrogen) and 19\% Polyethylene glycol PEG. Directly, 
fragments were sequenced in the two directions using the BigDye ${ }^{\mathrm{TM}}$ Terminator v3.1 Cycle Sequencing Kit (Thermo Fisher) in a capillary sequencer Applied Biosystems 3730xI DNA Analyzer (Thermo Fisher). FASTA sequences generated by mitogenome mapping and Sanger sequencing were then aligned and checked for differences.

\section{Genome-wide variation and analysis of divergence}

We examined levels of genetic diversity and differentiation between host-adapted population of each Varroa species. To investigate differentiation among populations, we first measured the absolute divergence $D_{x y}$ which requires both variants and invariants sites (Cruickshank and Hahn 2014). We also investigated levels of standing genetic variation $(\pi)$. For both analyses genome scans were computed using a sliding window method on the "all-sites dataset" (50kb window, sliding every $20 \mathrm{~kb}$ and containing a minimum of 100 SNPs). This method was implemented using the parseVCF.py and popgenWindow.py python scripts (github.com/simonhmartin/genomics_general) (Martin, Davey, and Jiggins 2015).

Using the biallelic "SNP-only dataset", we also estimated the genome-wide differentiation by calculating Weir and Cockerham's $F_{S T}$ per site using VCFtools. Subsequently, we assessed the genetic structure within species using a principal component analysis (PCA) performed with the R package vcfR (Knaus and Grünwald 2017). To further investigate the population structure and related ancestry, we conducted an incremental K step analysis using NGSadmix (Skotte, Korneliussen, and Albrechtsen 2013). We reduce the effect of linkage disequilibrium between SNPs by conducting a pruning using PLINK v1.90b3 (Purcell et al. 2007) on the "SNP-only dataset" with the following parameters: -indep-pairwise 2010 0.5. After removing linked SNPs, the pruned dataset containing 1,276,602 SNPs was converted into BEAGLE format and directly used in NGSadmix. A total of 10 replicates per K, from 2 to 20 , were run and the model with the highest likelihood was selected for plotting each $\mathrm{K}$. The number of genetic clusters among and within species was determined following guidelines in (Meirmans 2015; Lawson, van Dorp, and Falush 2018) and making biological sense.

\section{Mutation rate estimation from mother-son pairs}

Coalescent analysis requires an estimation of mutation rate, yet none is available for closely related species. To do this we collected and sequenced six $V$. destructor diploid mothers and their haploid sons for this purpose. These samples were collected from individual capped cells containing $A$. mellifera redeye drone pupae. Each family was selected under the following conditions: 1) each cell was only infected by a single mother-foundress mite, 2) a Varroa family at this stage should contained three to four offspring, including one haploid son and two to three diploid daughters, 3) the son was at the deutonymph or adult phase to avoid misidentification with protonymph sisters. We collected three families and preserved in absolute ethanol in February 2018 (\#1, \#2, and \#7), and three more in May 2018 (\#15, \#17, and \#19) from the same colony at the OIST Ecology and Evolution experimental apiary. 
Libraries were prepared using an NEBNext ${ }^{\circledR}$ Ultra ${ }^{\mathrm{TM}}$ II FS DNA Library Prep Kit (New England Biolabs, Inc) with a fragmentation size incubation time of $15 \mathrm{~min}$ at $37^{\circ} \mathrm{C}$, and three PCR cycles. Libraries were cleaned up using CA-magnetic beads (Dynabeads ${ }^{\circledR}$ MyOne Carboxylic Acid, Invitrogen), and 17\% PEG 6000 (Sigma-Aldrich ( $\mathrm{LLC}$ ). Libraries were pooled and sequenced on two lanes of HiSeq 4000 (Illumina $\circledR$ ) at the OIST sequencing center.

Mutation rate was estimated from GATK variant calls (McKenna et al. 2010) using DeNovoGear's dng-call algorithm (Ramu et al. 2013) which can model Varroa's haplodiploidy sex-determination system. Singlenucleotide mutations were called on the 7 largest contigs in the Varroa genome, avoiding sites that were within $100 \mathrm{bp}$ of an indel. Mutation calls were de-duplicated, retaining only sites that were biallelic and not part of a mutation cluster (with $100 \mathrm{bp}$ of another call or part of a long-run of calls from the same sample). After deduplication, the remaining calls were filtered such that $\mathrm{P}$ (denovol data) was high (DNP $>=0.75$ ) and the fit to the model was good (LLS >= -3). DNP (de-novo probability) and LLS (log-likelihood scaled) are both per-site statistics generated by dng-call. The denominator for the mutation rate analysis was estimated by simulating mutations at 1,000 locations in each son and calculating what fraction of these simulated mutations was recovered by our pipeline. A VCF was generated for these locations, and mutations were simulated by changing the son's haplotype to one of the other three bases. ALT and AD fields were updated as needed.

\section{Demographic analyses of host switch using SFS}

We inferred the demographic history of both $V$. destructor and $V$. jacobsoni mites with the coalescent simulator fastsimcoal v2.6 using the site frequency spectrum (SFS) (Excoffier et al. 2013). Demographic inferences were computed independently for each species and samples were subsetted following genome-wide analysis results while considering geographical sympatry and continuity. For $V$. destructor $(N=27)$, we excluded Nepal and the Japanese mites (continental island) whereas for $V$. jacobsoni $(N=12)$, we included only mites from Papua New Guinea (host switch region). To reduce the effect of selection which can biais the SFS (71), we kept SNPs and invariants from the "all-sites dataset" that were at least $50 \mathrm{~kb}$ away from any annotated genes regions (VCFtools exclude-bed option). The 2Djoint folded-SFS was computed for each species/host population on the filtered 12,594,802 sites including 224,568 SNPs, using the $v c f 2 s f s$ R scripts (github.com/shenglin-liu/vcf2sfs) (S. Liu et al. 2018).

We considered a scenario consistent with the known history of Varroa jumps (Figure S3). We incorporated the following demographic parameters (Table 2): the estimated effective population size for Varroa mites parasitizing $A$. cerana $\mathrm{N}_{\mathrm{VAC} 1}$ (in haploid genomes) to be stable before, during, and after the host switch. On the other hand, the mite population size on the new host $A$. mellifera expanded from $\mathrm{N}_{\text {BOTAM }}$ or not with a growth rate $\mathrm{G}_{\mathrm{AM}}$ after the host switch to reach modern population size $\mathrm{N}_{\text {VAMO }}$ (in haploid genomes). The host switch founder event occurred at a time $T_{\text {JUMP }}$ (in generations) and ended at a time $T_{\text {BOTEND }}$ (in generations). Finally, in a case of bidirectional migrations due to sympatry we 
estimated $M_{A M t o A C} / M_{A C t o A M}$, to be the proportion of haploid genomes migrating from one population to another.

We ran 100 replicates using the observed SFS as follow: a minimum of 20 loops (--minnumloops 20) and a maximum of 150 loops (e.g., ECM cycles, -numloops 100) were performed to estimate the parameters, with one million coalescent simulations per loop (--numsims 1,000,000), and a 0.001 minimum relative difference in parameter values estimated by the maximum composite likelihood (--maxlhood 0.001). The replicate with the highest likelihood set of estimated parameters was retained for model comparison.

To ensure that the upper limit genome-wide mutation rate $\mu=8.0 \times 10^{-10}$ previously used was adequate to estimate parameters from the observed SFS, we tested different $\mu$ levels. For this we ran scenario 4 in the same conditions as for scenario choice but $\mu$ ranging from $8.0 \times 10^{-10}$ to $1.0 \times 10^{-11}$. Additionally, fastsimcoal2.6 offers the possibility to input inbreeding coefficient. As Varroa populations showed high inbreeding, we also ran scenario 4 with $(F=0.7$, preliminary tests also ran with 0.8 and 0.9$)$ and without inbreeding coefficient. For all these conditions combined, we ran 100 replicates for each of the 10 combinations per species.

Finally, the overall best estimate parameters set was used to generate 100 pseudo-observed SFS (for a similar number of polymorphic SNPs) for parametric bootstraps. We repeated the parameters estimation for each pseudo-observed SFS and kept the best run. The top 100 runs estimated parameters values were used to calculate $95 \%$ confidence intervals. The mean and 95 percentile confidence intervals were computed using the 'boot' R package (Canty and Ripley 2019). Finally, the fit of the best expected SFS was visualized against the observed using SFStools R scripts (github.com/marqueda/SFS-scripts).

\section{Declarations}

\section{Acknowledgments}

MAT's research was supported by a postdoctoral fellowship from the Japan Society for Promotion of Science (JSPS) (P19723), Kakenhi Grant-in-Aid, (19F19723) and the Okinawa Institute of Science and Technology (OIST). ASM was supported by a Future Fellowship from the Australian Research Council (FT160100178) and a Kakenhi Grant-in-Aid for Scientific Research from the JSPS (18H02216). JMKR was supported by the Australian Centre for International Agricultural Research (ACIAR) and the Australian Department of Agriculture, Water and Environment (DAWE). RAC was supported by National Institutes of Health (R01-HG007178). We are grateful to Jo Si Lay Tan and Lijun Qiu for their advice and guidance in developing the wet lab workflow for Varroa mite sequencing. We also would like to thank the OIST Sequencing Center for assisting us in the sample sequencing. We wish to thank Steven D. Aird, technical editor, for reviewing and improving our manuscript.

\section{Author Contributions}


MAT and ASM designed research, analyzed population genetics data and wrote the manuscript. MAT processed the samples in the wet lab until library preparation and ran the demographic inferences. JMKR collected and provided samples from the mite CSIRO collection, and helped in the data interpretation. RAC analyzed data, wrote the manuscript, provided reproducible online resources and data for the estimation of mutation rate.

\section{Competing interests}

The authors declare that they have no competing interests.

\section{Data accessibility}

Raw FASTQ files for all individuals sequenced were submitted to the DNA Databank of Japan and uploaded to GenBank under the bioproject PRJDB9195 with the run accessions DRR209082-DRR209125 and DRR212369-DRR212380. The pipeline developed using Snakemake, Rmarkdown for data exploration, demographic inferences input files and FASTA sequences alignment (mtDNA) are made available for reproducibility on https://github.com/MaevaTecher/varroa-host-jump. Variant calling files, genome indexing files and input lists are readily available at DRYAD.

\section{Ethics approval and consent to participate}

Not applicable.

\section{Consent for publication}

Not applicable.

\section{References}

1. Anderson, D. L., and J. W. Trueman. 2000. "Varroa Jacobsoni (Acari: Varroidae) Is More than One Species." Experimental \& Applied Acarology 24 (3): 165-89.

2. Anderson, Denis L., and Stefan Fuchs. 1998. "Two Genetically Distinct Populations of Varroa Jacobsoni with Contrasting Reproductive Abilities on Apis Mellifera." Journal of Apicultural Research 37 (2): 69-78.

3. Andrews, Simon, and Others. 2010. "FastQC: A Quality Control Tool for High Throughput Sequence Data."

4. Araujo, Sabrina B. L., Mariana Pires Braga, Daniel R. Brooks, Salvatore J. Agosta, Eric P. Hoberg, Francisco W. von Hartenthal, and Walter A. Boeger. 2015. "Understanding Host-Switching by Ecological Fitting." PloS One 10 (10): e0139225.

5. Auger-Rozenberg, M-A, T. Boivin, E. Magnoux, C. Courtin, A. Roques, and C. Kerdelhué. 2012. "Inferences on Population History of a Seed Chalcid Wasp: Invasion Success despite a Severe Founder Effect from an Unexpected Source Population." Molecular Ecology 21 (24): 6086-6103. 
6. Beaurepaire, Alexis L., Arrigo Moro, Fanny Mondet, Yves Le Conte, Peter Neumann, and Barbara Locke. 2019. "Population Genetics of Ectoparasitic Mites Suggest Arms Race with Honeybee Hosts." Scientific Reports 9 (1): 11355.

7. Beaurepaire, Alexis L., Tuan A. Truong, Alejandro C. Fajardo, Tam Q. Dinh, Cleofas Cervancia, and Robin F. A. Moritz. 2015. "Host Specificity in the Honeybee Parasitic Mite, Varroa Spp. in Apis Mellifera and Apis Cerana." PloS One 10 (8): e0135103.

8. Canty, Angelo, and Brian Ripley. 2019. “Package 'Boot."” Version. ftp://ftp.math.ethz.ch/sfs/RCRAN/web/packages/boot/boot.pdf.

9. Chantawannakul, Panuwan, Lilia I. de Guzman, Jilian Li, and Geoffrey R. Williams. 2016. "Parasites, Pathogens, and Pests of Honeybees in Asia." Apidologie 47 (3): 301-24.

10. Chen, Chao, Huihua Wang, Zhiguang Liu, Xiao Chen, Jiao Tang, Fanming Meng, and Wei Shi. 2018. "Population Genomics Provide Insights into the Evolution and Adaptation of the Eastern Honey Bee (Apis Cerana)." Molecular Biology and Evolution 35 (9): 2260-71.

11. Cornuet, J. M., M. A. Beaumont, A. Estoup, and M. Solignac. 2006. "Inference on Microsatellite Mutation Processes in the Invasive Mite, Varroa Destructor, Using Reversible Jump Markov Chain Monte Carlo." Theoretical Population Biology 69 (2): 129-44.

12. Crane, Eva. 1968. "Beekeeping Round the World." Bee World 49 (3): 113-14.

13. Crozier, R. H., and Y. C. Crozier. 1993. "The Mitochondrial Genome of the Honeybee Apis Mellifera: Complete Sequence and Genome Organization." Genetics 133 (1): 97-117.

14. Cruickshank, Tami E., and Matthew W. Hahn. 2014. "Reanalysis Suggests That Genomic Islands of Speciation Are Due to Reduced Diversity, Not Reduced Gene Flow." Molecular Ecology 23 (13): 313357.

15. Danecek, Petr, Adam Auton, Goncalo Abecasis, Cornelis A. Albers, Eric Banks, Mark A. DePristo, Robert E. Handsaker, et al. 2011. "The Variant Call Format and VCFtools." Bioinformatics 27 (15): 2156-58.

16. Demastes, James W., David J. Hafner, Mark S. Hafner, Jessica E. Light, and Theresa A. Spradling. 2019. "Loss of Genetic Diversity, Recovery and Allele Surfing in a Colonizing Parasite, Geomydoecus Aurei." Molecular Ecology 28 (4): 703-20.

17. Dietemann, Vincent, Alexis Beaurepaire, Paul Page, Orlando Yañez, Ninat Buawangpong, Panuwan Chantawannakul, and Peter Neumann. 2019. "Population Genetics of Ectoparasitic Mites Varroa Spp. in Eastern and Western Honey Bees." Parasitology 146 (11): 1429-39.

18. Dietemann, Vincent, Francesco Nazzi, Stephen J. Martin, Denis L. Anderson, Barbara Locke, Keith S. Delaplane, Quentin Wauquiez, et al. 2013. "Standard Methods for Varroa Research." Journal of Apicultural Research 52 (1): 1-54.

19. Elbeaino, Toufic, Nouraldin Daher-Hjaij, Faiz Ismaeil, Jamal Mando, Bassem Solaiman Khaled, and Raied Abou Kubaa. 2016. "Occurrence of Deformed Wing Virus, Chronic Bee Paralysis Virus and MtDNA Variants in Haplotype K of Varroa Destructor Mites in Syrian Apiaries." Experimental \& Applied Acarology 69 (1): 11-19. 
20. Evans, Jay D., and Steven C. Cook. 2018. "Genetics and Physiology of Varroa Mites." Current Opinion in Insect Science 26 (April): 130-35.

21. Excoffier, Laurent, Isabelle Dupanloup, Emilia Huerta-Sánchez, Vitor C. Sousa, and Matthieu Foll. 2013. "Robust Demographic Inference from Genomic and SNP Data." PLoS Genetics 9 (10): e1003905.

22. Fazalova, Varvara, and Bruno Nevado. 2020. "Low Spontaneous Mutation Rate and Pleistocene Radiation of Pea Aphids." Molecular Biology and Evolution 37 (7): 2045-51.

23. Feder, Jeffrey L., Scott P. Egan, and Patrik Nosil. 2012. "The Genomics of Speciation-with-Gene-Flow." Trends in Genetics: TIG 28 (7): 342-50.

24. Fry, William E. 2016. "Phytophthora Infestans: New Tools (and Old Ones) Lead to New Understanding and Precision Management." Annual Review of Phytopathology 54 (1): 529-47.

25. Gajic, Bojan, Zeljko Radulovic, Jevrosima Stevanovic, Zoran Kulisic, Milos Vucicevic, Predrag Simeunovic, and Zoran Stanimirovic. 2013. "Variability of the Honey Bee Mite Varroa Destructor in Serbia, Based on MtDNA Analysis." Experimental \& Applied Acarology 61 (1): 97-105.

26. Garrison, Erik, and Gabor Marth. 2012. "Haplotype-Based Variant Detection from Short-Read Sequencing." ArXiv [q-Bio.GN]. arXiv. http://arxiv.org/abs/1207.3907.

27. González-Cabrera, Joel, Sonia Rodríguez-Vargas, T. G. Emyr Davies, Linda M. Field, Daniel Schmehl, James D. Ellis, Klemens Krieger, and Martin S. Williamson. 2016. "Novel Mutations in the VoltageGated Sodium Channel of Pyrethroid-Resistant Varroa Destructor Populations from the Southeastern USA." PloS One 11 (5): e0155332.

28. Guzman, Lilia I. de, Thomas E. Rinderer, and J. Anthony Stelzer. 1999. "Occurrence of Two Genotypes of Varroa Jacobsoni Oud. in North America." Apidologie 30 (1): 31-36.

29. Harada, Reona, Masato Yoshioka, Hisashi Okuyama, Manabu Kato, Stephen J. Martin, and Jun-Ichi Takahashi. 2020. "Complete Mitochondrial DNA Sequence of the Parasitic Honey Bee Mite Varroa Destructor (Mesostigmata: Varroidae)." Mitochondrial DNA. Part B, Resources 5 (1): 635-36.

30. Hatcher, Melanie J., Jaimie T. A. Dick, and Alison M. Dunn. 2012. “Disease Emergence and Invasions." Functional Ecology 26 (6): 1275-87.

31. Huson, Daniel H., and David Bryant. 2006. "Application of Phylogenetic Networks in Evolutionary Studies." Molecular Biology and Evolution 23 (2): 254-67.

32. Ilyasov, Rustem A., Han Gi Youn, Myeong-Lyeol Lee, Kil Won Kim, Maxim Y. Proshchalykin, Arkady S. Lelej, Jun-Ichi Takahashi, and Hyung Wook Kwon. 2019. "Phylogenetic Relationships of Russian FarEast Apis Cerana with Other North Asian Populations." Journal of Apicultural Science 63 (2): 289314.

33. Ji, Yongkun, Xingan Li, Ting Ji, Junbo Tang, Lifei Qiu, Jiahui Hu, Jiangxing Dong, et al. 2020. “Gene Reuse Facilitates Rapid Radiation and Independent Adaptation to Diverse Habitats in the Asian Honeybee." Science Advances 6 (51): eabd3590.

34. Knaus, Brian J., and Niklaus J. Grünwald. 2017. "Vcfr: A Package to Manipulate and Visualize Variant Call Format Data in R." Molecular Ecology Resources 17 (1): 44-53. 
35. Köster, Johannes, and Sven Rahmann. 2012. "Snakemake-a Scalable Bioinformatics Workflow Engine." Bioinformatics 28 (19): 2520-22.

36. Langmead, Ben, and Steven L. Salzberg. 2012. "Fast Gapped-Read Alignment with Bowtie 2." Nature Methods 9 (4): 357-59.

37. Lawson, Daniel J., Lucy van Dorp, and Daniel Falush. 2018. "A Tutorial on How Not to Over-Interpret STRUCTURE and ADMIXTURE Bar Plots." Nature Communications 9 (1): 3258.

38. Lenormand, Thomas. 2002. "Gene Flow and the Limits to Natural Selection." Trends in Ecology \& Evolution 17 (4): 183-89.

39. Letunic, Ivica, and Peer Bork. 2019. "Interactive Tree Of Life (ITOL) v4: Recent Updates and New Developments." Nucleic Acids Research 47 (W1): W256-59.

40. Li, Heng. 2011. "A Statistical Framework for SNP Calling, Mutation Discovery, Association Mapping and Population Genetical Parameter Estimation from Sequencing Data." Bioinformatics 27 (21): 2987-93.

41. Li, Heng, Bob Handsaker, Alec Wysoker, Tim Fennell, Jue Ruan, Nils Homer, Gabor Marth, Goncalo Abecasis, Richard Durbin, and 1000 Genome Project Data Processing Subgroup. 2009. "The Sequence Alignment/Map Format and SAMtools." Bioinformatics 25 (16): 2078-79.

42. Li, Wenfeng, Cheng Wang, Zachary Y. Huang, Yanping Chen, and Richou Han. 2019. "Reproduction of Distinct Varroa Destructor Genotypes on Honey Bee Worker Brood." Insects 10 (11): 372.

43. Liu, Haoxuan, Yanxiao Jia, Xiaoguang Sun, Dacheng Tian, Laurence D. Hurst, and Sihai Yang. 2017. "Direct Determination of the Mutation Rate in the Bumblebee Reveals Evidence for Weak Recombination-Associated Mutation and an Approximate Rate Constancy in Insects." Molecular Biology and Evolution 34 (1): 119-30.

44. Liu, Shenglin, Anne-Laure Ferchaud, Peter Grønkjaer, Rasmus Nygaard, and Michael M. Hansen. 2018. "Genomic Parallelism and Lack Thereof in Contrasting Systems of Three-Spined Sticklebacks." Molecular Ecology 27 (23): 4725-43.

45. Martin, Simon H., John W. Davey, and Chris D. Jiggins. 2015. "Evaluating the Use of ABBA-BABA Statistics to Locate Introgressed Loci." Molecular Biology and Evolution 32 (1): 244-57.

46. McDonald, Bruce A., and Celeste Linde. 2002. "Pathogen Population Genetics, Evolutionary Potential, and Durable Resistance." Annual Review of Phytopathology 40 (February): 349-79.

47. McKenna, Aaron, Matthew Hanna, Eric Banks, Andrey Sivachenko, Kristian Cibulskis, Andrew Kernytsky, Kiran Garimella, et al. 2010. "The Genome Analysis Toolkit: A MapReduce Framework for Analyzing next-Generation DNA Sequencing Data." Genome Research 20 (9): 1297-1303.

48. McMullan, Mark, Maryam Rafiqi, Gemy Kaithakottil, Bernardo J. Clavijo, Lorelei Bilham, Elizabeth Orton, Lawrence Percival-Alwyn, et al. 2018. "The Ash Dieback Invasion of Europe Was Founded by Two Genetically Divergent Individuals." Nature Ecology \& Evolution 2 (6): 1000-1008.

49. Meirmans, Patrick G. 2015. "Seven Common Mistakes in Population Genetics and How to Avoid Them." Molecular Ecology 24 (13): 3223-31. 
50. Navajas, Maria, Denis L. Anderson, Lilia I. de Guzman, Zachary Y. Huang, Jeremy Clement, Ting Zhou, and Yves Le Conte. 2010. "New Asian Types of Varroa Destructor: A Potential New Threat for World Apiculture." Apidologie 41 (2): 181-93.

51. Navajas, Maria, Y. Le Conte, M. Solignac, S. Cros-Arteil, and J-M Cornuet. 2002. “The Complete Sequence of the Mitochondrial Genome of the Honeybee Ectoparasite Mite Varroa Destructor (Acari: Mesostigmata)." Molecular Biology and Evolution 19 (12): 2313-17.

52. Nguyen, Lam-Tung, Heiko A. Schmidt, Arndt von Haeseler, and Bui Quang Minh. 2015. "IQ-TREE: A Fast and Effective Stochastic Algorithm for Estimating Maximum-Likelihood Phylogenies." Molecular Biology and Evolution 32 (1): 268-74.

53. Noël, Amélie, Yves Le Conte, and Fanny Mondet. 2020. “Varroa Destructor: How Does It Harm Apis Mellifera Honey Bees and What Can Be Done about It?" Emerging Topics in Life Sciences 4 (1): 4557.

54. Nylin, Sören, Salvatore Agosta, Staffan Bensch, Walter A. Boeger, Mariana P. Braga, Daniel R. Brooks, Matthew L. Forister, et al. 2018. “Embracing Colonizations: A New Paradigm for Species Association Dynamics." Trends in Ecology \& Evolution 33 (1): 4-14.

55. Ogihara, Mari H., Mikio Yoshiyama, Nobuo Morimoto, and Kiyoshi Kimura. 2020. “Dominant Honeybee Colony Infestation by Varroa Destructor (Acari: Varroidae) K Haplotype in Japan." Applied Entomology and Zoology 55 (2): 189-97.

56. Penczykowski, Rachel M., Anna-Liisa Laine, and Britt Koskella. 2016. "Understanding the Ecology and Evolution of Host-Parasite Interactions across Scales." Evolutionary Applications 9 (1): 37-52.

57. Pinho, Catarina, and Jody Hey. 2010. “Divergence with Gene Flow: Models and Data." Annual Review of Ecology, Evolution, and Systematics 41 (1): 215-30.

58. Posada-Florez, Francisco, Daniel E. Sonenshine, Noble I. Egekwu, Clifford Rice, Robert Lupitskyy, and Steven C. Cook. 2019. "Insights into the Metabolism and Behaviour of Varroa Destructor Mites from Analysis of Their Waste Excretions." Parasitology 146 (4): 527-32.

59. Poulin, Robert, Boris R. Krasnov, David Mouillot, and David W. Thieltges. 2011. "The Comparative Ecology and Biogeography of Parasites." Philosophical Transactions of the Royal Society of London. Series B, Biological Sciences 366 (1576): 2379-90.

60. Purcell, Shaun, Benjamin Neale, Kathe Todd-Brown, Lori Thomas, Manuel A. R. Ferreira, David Bender, Julian Maller, et al. 2007. “PLINK: A Tool Set for Whole-Genome Association and Population-Based Linkage Analyses." American Journal of Human Genetics 81 (3): 559-75.

61. Radloff, Sarah E., Colleen Hepburn, H. Randall Hepburn, Stefan Fuchs, Soesilawati Hadisoesilo, Ken Tan, Michael S. Engel, and Viktor Kuznetsov. 2010. "Population Structure and Classification of Apis Cerana." Apidologie 41 (6): 589-601.

62. Ramsey, Samuel D., Ronald Ochoa, Gary Bauchan, Connor Gulbronson, Joseph D. Mowery, Allen Cohen, David Lim, et al. 2019. "Varroa Destructor Feeds Primarily on Honey Bee Fat Body Tissue and Not Hemolymph." Proceedings of the National Academy of Sciences of the United States of America 116 (5): 1792-1801. 
63. Ramu, Avinash, Michiel J. Noordam, Rachel S. Schwartz, Arthur Wuster, Matthew E. Hurles, Reed A. Cartwright, and Donald F. Conrad. 2013. “DeNovoGear: De Novo Indel and Point Mutation Discovery and Phasing." Nature Methods 10 (10): 985-87.

64. Rigaud, Thierry, Marie-Jeanne Perrot-Minnot, and Mark J. F. Brown. 2010. “Parasite and Host Assemblages: Embracing the Reality Will Improve Our Knowledge of Parasite Transmission and Virulence." Proceedings. Biological Sciences / The Royal Society 277 (1701): 3693-3702.

65. Roberts, J. M. K., D. L. Anderson, and W. T. Tay. 2015. “Multiple Host Shifts by the Emerging Honeybee Parasite, Varroa Jacobsoni.” Molecular Ecology 24 (10): 2379-91.

66. Robinson, John D., Alec J. Coffman, Michael J. Hickerson, and Ryan N. Gutenkunst. 2014. "Sampling Strategies for Frequency Spectrum-Based Population Genomic Inference." BMC Evolutionary Biology 14 (December): 254.

67. Rueppell, O., A. M. Hayes, N. Warrit, and D. R. Smith. 2011. “Population Structure of Apis Cerana in Thailand Reflects Biogeography and Current Gene Flow Rather than Varroa Mite Association." Insectes Sociaux 58 (4): 445-52.

68. Sedlazeck, Fritz J., Philipp Rescheneder, and Arndt von Haeseler. 2013. “NextGenMap: Fast and Accurate Read Mapping in Highly Polymorphic Genomes.” Bioinformatics 29 (21): 2790-91.

69. Sexton, Jason P., Jorge Montiel, Jackie E. Shay, Molly R. Stephens, and Rachel A. Slatyer. 2017. "Evolution of Ecological Niche Breadth." Annual Review of Ecology, Evolution, and Systematics 48 (1): 183-206.

70. Skotte, Line, Thorfinn Sand Korneliussen, and Anders Albrechtsen. 2013. “Estimating Individual Admixture Proportions from next Generation Sequencing Data.” Genetics 195 (3): 693-702.

71. Solignac, Michel, Jean-Marie Cornuet, Dominique Vautrin, Yves Le Conte, Denis Anderson, Jay Evans, Sandrine Cros-Arteil, and Maria Navajas. 2005. "The Invasive Korea and Japan Types of Varroa Destructor, Ectoparasitic Mites of the Western Honeybee (Apis Mellifera), Are Two Partly Isolated Clones." Proceedings. Biological Sciences / The Royal Society 272 (1561): 411-19.

72. Tan, Hong-Wei, Guo-Hua Liu, Xia Dong, Rui-Qing Lin, Hui-Qun Song, Si-Yang Huang, Zi-Guo Yuan, Guang-Hui Zhao, and Xing-Quan Zhu. 2011. "The Complete Mitochondrial Genome of the Asiatic Cavity-Nesting Honeybee Apis Cerana (Hymenoptera: Apidae).” PloS One 6 (8): e23008.

73. Tan, Ken, Yufeng Qu, Zhengwei Wang, Zhiwei Liu, and Michael S. Engel. 2016. “Haplotype Diversity and Genetic Similarity among Populations of the Eastern Honey Bee from Himalaya-Southwest China and Nepal (Hymenoptera: Apidae)." Apidologie 47 (2): 197-205.

74. Techer, Maeva A., Rahul V. Rane, Miguel L. Grau, John M. K. Roberts, Shawn T. Sullivan, Ivan Liachko, Anna K. Childers, Jay D. Evans, and Alexander S. Mikheyev. 2019. “Divergent Evolutionary Trajectories Following Speciation in Two Ectoparasitic Honey Bee Mites." Communications Biology 2 (1): 357.

75. Tomasini, Matteo, and Stephan Peischl. 2020. "When Does Gene Flow Facilitate Evolutionary Rescue?" Evolution; International Journal of Organic Evolution 74 (8): 1640-53. 
76. Traynor, Kirsten S., Fanny Mondet, Joachim R. de Miranda, Maeva Techer, Vienna Kowallik, Melissa A. Y. Oddie, Panuwan Chantawannakul, and Alison McAfee. 2020. "Varroa Destructor: A Complex Parasite, Crippling Honey Bees Worldwide." Trends in Parasitology 36 (7): 592-606.

77. Wala, Jeremiah, Cheng-Zhong Zhang, Matthew Meyerson, and Rameen Beroukhim. 2016. "VariantBam: Filtering and Profiling of next-Generational Sequencing Data Using Region-Specific Rules." Bioinformatics 32 (13): 2029-31.

78. Warrit, Natapot, Deborah Roan Smith, and Chariya Lekprayoon. 2006. “Genetic Subpopulations of Varroa Mites and Their Apis Cerana Hosts in Thailand." Apidologie 37 (1): 19-30.

79. Whiteley, Andrew R., Sarah W. Fitzpatrick, W. Chris Funk, and David A. Tallmon. 2015. "Genetic Rescue to the Rescue." Trends in Ecology \& Evolution 30 (1): 42-49.

80. Yamasaki, Yo Y., Ryo Kakioka, Hiroshi Takahashi, Atsushi Toyoda, Atsushi J. Nagano, Yoshiyasu Machida, Peter R. Møller, and Jun Kitano. 2020. "Genome-Wide Patterns of Divergence and Introgression after Secondary Contact between Pungitius Sticklebacks." Philosophical Transactions of the Royal Society of London. Series B, Biological Sciences 375 (1806): 20190548.

81. Yancan, Li, Chao Tianle, Fan Yunhan, Lou Delong, and Wang Guizhi. 2019. "Population Genomics and Morphological Features Underlying the Adaptive Evolution of the Eastern Honey Bee (Apis Cerana)." BMC Genomics 20 (1): 869.

82. Zhou, Ting, Denis L. Anderson, Zachary Y. Huang, Shuangxiu Huang, Jun Yao, Tan Ken, and Qingwen Zhang. 2004. "Identification of Varroa Mites (Acari: Varroidae) Infesting Apis Cerana and Apis Mellifera in China." Apidologie 35 (6): 645-54.

\section{Tables}

Table 1: Varroa specimens used for population genomics were collected from their native range across 11 countries, both on their original or new honey bee host. Newly reported mtDNA lineages are indicated in bold 


\begin{tabular}{|c|c|c|c|c|c|}
\hline Species & Host & Country & Date & $\mathbf{N}$ & mtDNA lineages as defined by standard $C O X 1$ \\
\hline \multirow[t]{7}{*}{ V. destructor } & $\begin{array}{l}\text { Original host } \\
\text { A. cerana }\end{array}$ & Japan & 1994 & 2 & VD Japan J1 \\
\hline & & South Korea & 1996 & 1 & VD Korea K1 \\
\hline & & China & 2001-2002 & 6 & $\begin{array}{l}\text { VD China C1 } \\
\text { VD China C3 } \\
\text { VD China C4 } \\
\text { VD Viet Nam V1 }\end{array}$ \\
\hline & & Thailand & 2003 & 1 & VD Viet Nam V1 \\
\hline & & Viet Nam & 1996 & 2 & $\begin{array}{l}\text { VD Viet Nam V1 } \\
\text { VD Korea K1 }\end{array}$ \\
\hline & & Myanmar & 2003 & 1 & VD Viet Nam V1 \\
\hline & & Nepal & 2000 & 1 & VD Nepal 2 \\
\hline \multirow[t]{5}{*}{ V. destructor } & $\begin{array}{l}\text { New host } \\
\text { A. mellifera }\end{array}$ & Japan & 1996 & 1 & VD Korea K1 \\
\hline & & South Korea & 1996 & 3 & VD Korea K1 \\
\hline & & China & 2001 & 5 & VD Korea K1 \\
\hline & & Viet Nam & 1996, 1998 & 6 & VD Korea K1 \\
\hline & & Myanmar & 2003 & 2 & VD Korea K1 \\
\hline \multirow[t]{5}{*}{ V. jacobsoni } & $\begin{array}{l}\text { Original host } \\
\text { A. cerana }\end{array}$ & India & 2002 & 2 & VJ Bengaluru 1 \\
\hline & & Thailand & 2003 & 1 & VJ Laos L1 \\
\hline & & Viet Nam & 2003-2004 & 5 & VJ Laos L1 \\
\hline & & Malaysia & 1995 & 1 & VJ Malaysia 2 \\
\hline & & Indonesia & 1998,2002 & 6 & $\begin{array}{l}\text { VJ Java } 1 \\
\text { VJ Lombok } 2\end{array}$ \\
\hline
\end{tabular}




\begin{tabular}{|l|l|l|l|l|l|}
\hline & & Papua New Guinea & $1997,2008,2015$ & 4 & VJ PNG 1 \\
\hline V. jacobsoni & Original host & Indonesia & 1996 & 3 & VJ Java 1 \\
\hline V. jacobsoni & New host & Thailand & 1994 & 1 & VJ North Thailand 4 \\
& A. mellifera & & & & \\
\hline & & Papua New Guinea & 2008,2014 & 9 & VJ PNG 1 \\
\hline
\end{tabular}

Table 2: Parameter estimates for the most likely demographic scenarios with isolation with migration and population expansion for each species jump. As stated in fastsimcoal 2 manual, these upper-bound priors indicated with an asterisk were not bounded by the analysis. 


\begin{tabular}{|c|c|c|c|c|c|c|}
\hline & \multicolumn{2}{|c|}{ Priors } & \multicolumn{2}{|c|}{ V. destructor } & \multicolumn{2}{|c|}{ V. jacobsoni } \\
\hline & Distribution & Range & $\begin{array}{l}\text { Mean } \\
\text { value }\end{array}$ & CI 95\% & $\begin{array}{l}\text { Mean } \\
\text { value }\end{array}$ & CI 95\% \\
\hline $\begin{array}{l}\mathbf{N}_{\mathrm{VAC} 1} \\
\text { (haploid genomes) } \\
\text { At present, mite effective populatio } \\
\text { A. cerana }\end{array}$ & uniform & $\begin{array}{c}{[2 ;} \\
20,000 *]\end{array}$ & 17,166 & $\begin{array}{r}{[16,598 ;} \\
17,731]\end{array}$ & 17,376 & $\begin{array}{l}{[16589 ;} \\
18144]\end{array}$ \\
\hline $\begin{array}{l}\mathbf{N}_{\text {VAM0 }} \\
\text { (haploid genomes) } \\
\text { At present, mite effective populatio } \\
\text { mellifera }\end{array}$ & uniform & $\begin{array}{c}{[2 ;} \\
\left.20,000^{*}\right]\end{array}$ & 29,659 & $\begin{array}{r}{[28,722 ;} \\
30,666]\end{array}$ & 5,220 & $\begin{array}{l}{[4,787 ;} \\
5,683]\end{array}$ \\
\hline $\begin{array}{l}\mathbf{N}_{\text {BOTAM }} \\
\text { (haploid genomes) } \\
\text { Past mite effective founding popula } \\
\text { switch }\end{array}$ & uniform & $\begin{array}{c}{[2 ;} \\
\left.1,000^{*}\right]\end{array}$ & 156 & $\begin{array}{l}{[146 ;} \\
165]\end{array}$ & 138 & $\begin{array}{l}{[126 ;} \\
151]\end{array}$ \\
\hline $\begin{array}{l}\mathrm{T}_{\mathrm{JUMP}} \\
\text { (generation) } \\
\text { Time of new founding population sp }\end{array}$ & $\begin{array}{l}\text { uniform and } \\
\text { bounded }\end{array}$ & {$[1 ; 1,000]$} & 887 & $\begin{array}{l}{[866 ;} \\
906]\end{array}$ & 183 & $\begin{array}{l}{[179 ;} \\
187]\end{array}$ \\
\hline $\begin{array}{l}\mathrm{T}_{\text {BOTEND }} \\
\text { (generation) } \\
\text { End of the founder effect period }\end{array}$ & $\begin{array}{l}\text { uniform and } \\
\text { bounded }\end{array}$ & {$[1 ; 1,000]$} & 710 & $\begin{array}{l}{[693 ;} \\
726]\end{array}$ & 170 & $\begin{array}{l}{[165 ;} \\
175]\end{array}$ \\
\hline $\begin{array}{l}\mathrm{G}_{\mathrm{AM}} \\
\text { (haploid genomes per generation) } \\
\text { Growth rate of the expanding newly } \\
\text { population (from present to past) }\end{array}$ & uniform & {$[-1 ; 0 *]$} & -0.0014 & $\begin{array}{r}{[-0.0015 ;} \\
-0.0013]\end{array}$ & -0.4636 & $\begin{array}{r}{[-0.4895 ;} \\
-0.4399]\end{array}$ \\
\hline $\begin{array}{l}\mathbf{M}_{\text {ACtoAM }} \\
\text { (haploid genomes per generation) } \\
\text { Migration rate of parasite spillover } \\
\text { host) }\end{array}$ & $\log$ uniform & $\begin{array}{c}1 \times 10^{-6} \\
\left.100^{*}\right]\end{array}$ & 0.0003 & $\begin{array}{l}{[0.0003 ;} \\
0.0003]\end{array}$ & 0.0161 & $\begin{array}{l}{[0.0154 ;} \\
0.0168]\end{array}$ \\
\hline $\begin{array}{l}\mathbf{M}_{\mathrm{AMtoAC}} \\
\text { (haploid genomes per generation) }\end{array}$ & $\log$ uniform & $\begin{array}{c}{\left[1 \times 10^{-6} ;\right.} \\
\left.100^{*}\right]\end{array}$ & 0.0001 & $\begin{array}{l}{[0.0001 ;} \\
0.0001]\end{array}$ & 0.0295 & $\begin{array}{l}\text { [0.0283; } \\
0.0309]\end{array}$ \\
\hline
\end{tabular}




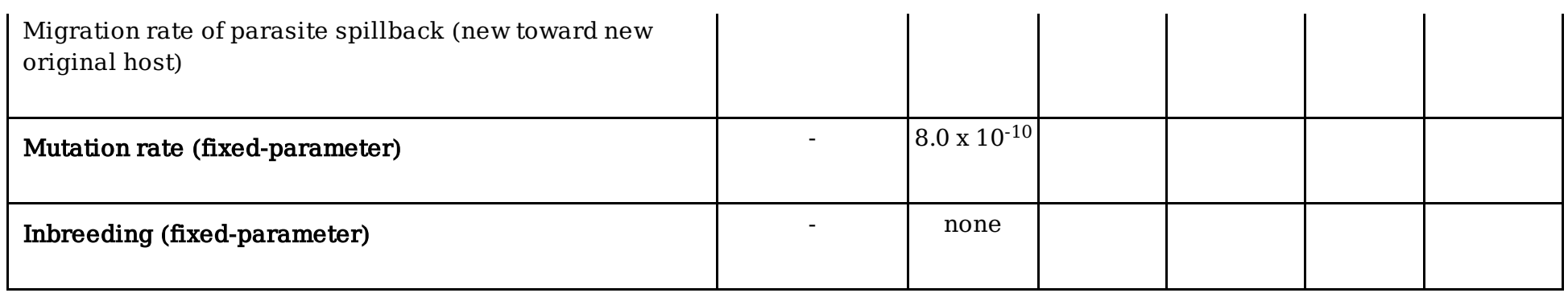

\section{Figures}

A

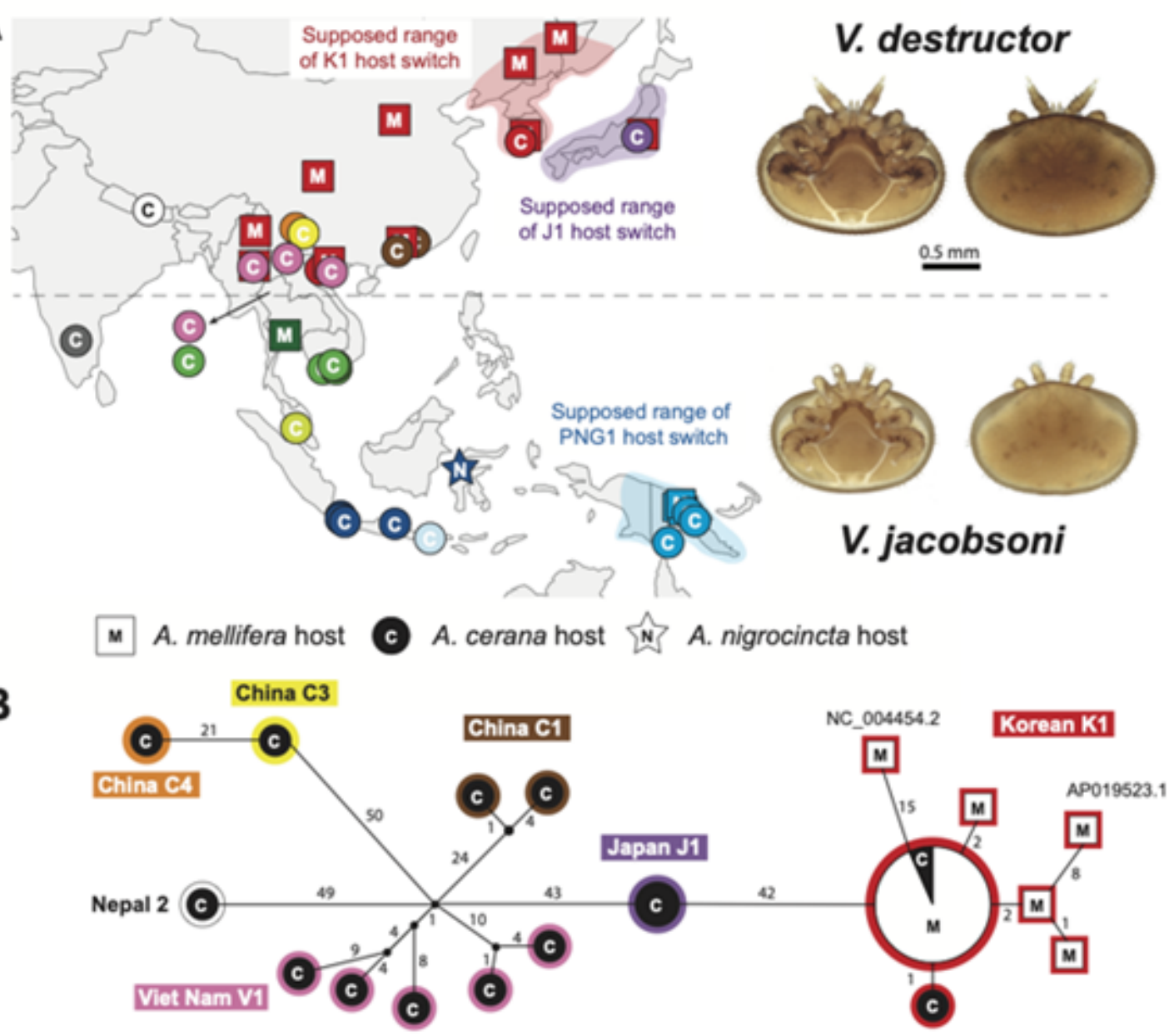

C

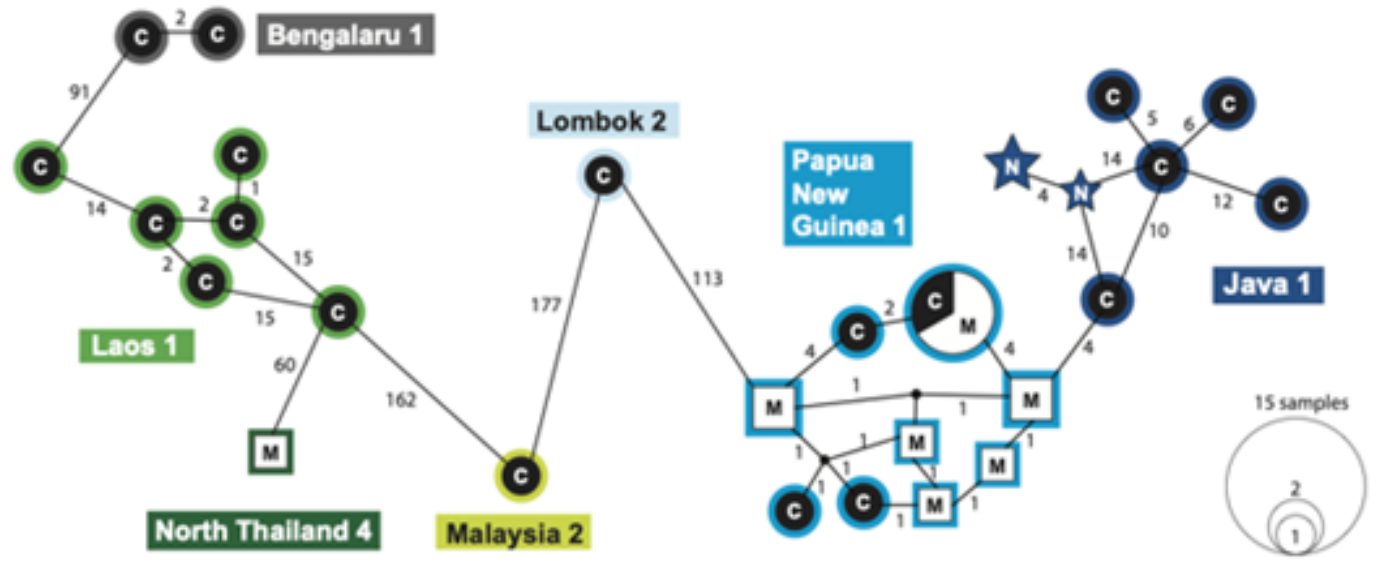




\section{Figure 1}

Mitogenomic phylogeographic networks uncover cryptic diversity in host-switched lineages. Both mite species exhibit geographic structure on their original host, with lineages classified using their COX1 sequences (A). The color of each circle/square indicates a unique mitogenome sequence and size is proportionate to the sample size (B). Previous studies based on COX1 found that V. destructor hostswitched lineages largely belong to the so-called 'Korean K1' haplotype. However, the mitogenome-wide analysis revealed four to six mitotypes on the new host, suggesting previously unreported switches by mites from the same geographic region (B). For the most recent jump in 2008, out the seven lineages identified for V. jacobsoni only the known PNG1 succeeded in crossing the species barrier (Roberts, Anderson, and Tay 2015) (C). Yet, looking at the whole mitochondrial genome, it becomes evident that the host switch involved a number of independent female founders from closely related populations, or even the same population. Thus, mitogenomic data suggest a relatively diverse population of host-switched mites in both species. Note: The designations employed and the presentation of the material on this map do not imply the expression of any opinion whatsoever on the part of Research Square concerning the legal status of any country, territory, city or area or of its authorities, or concerning the delimitation of its frontiers or boundaries. This map has been provided by the authors. 

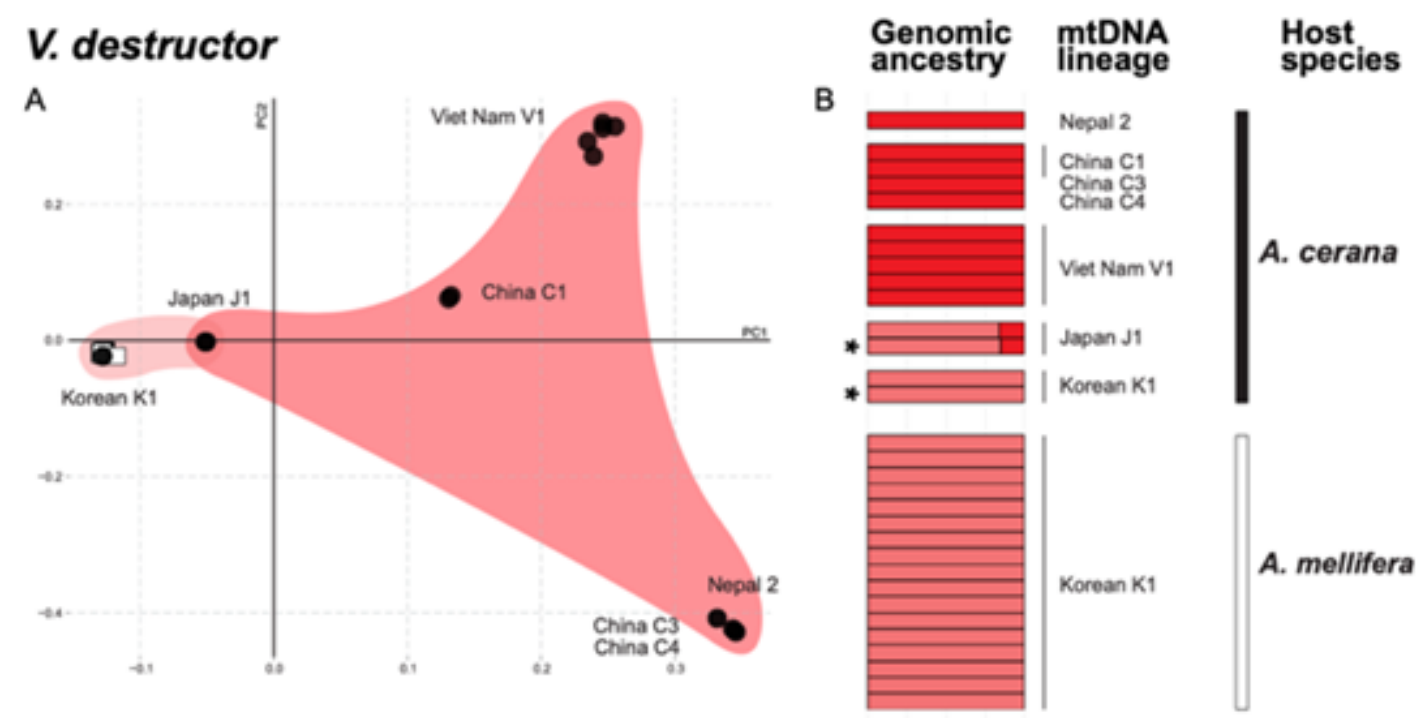

\section{V. jacobsoni}

C

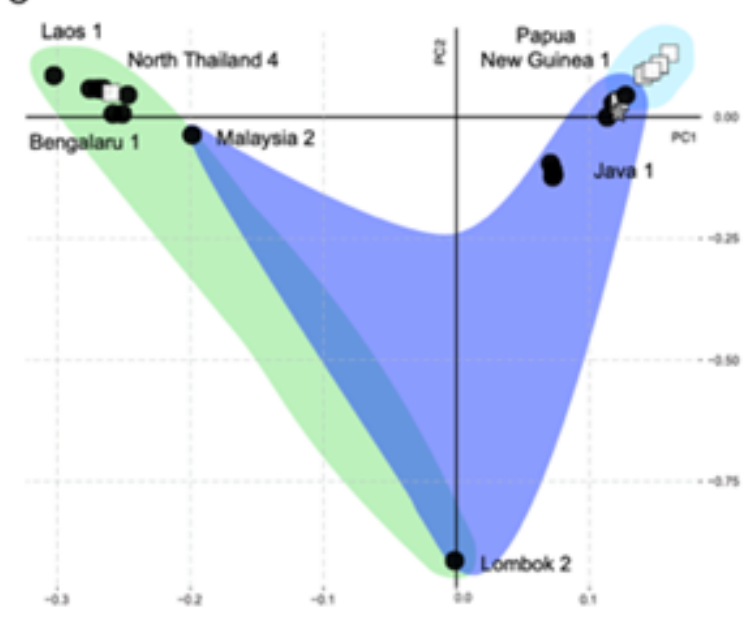

D

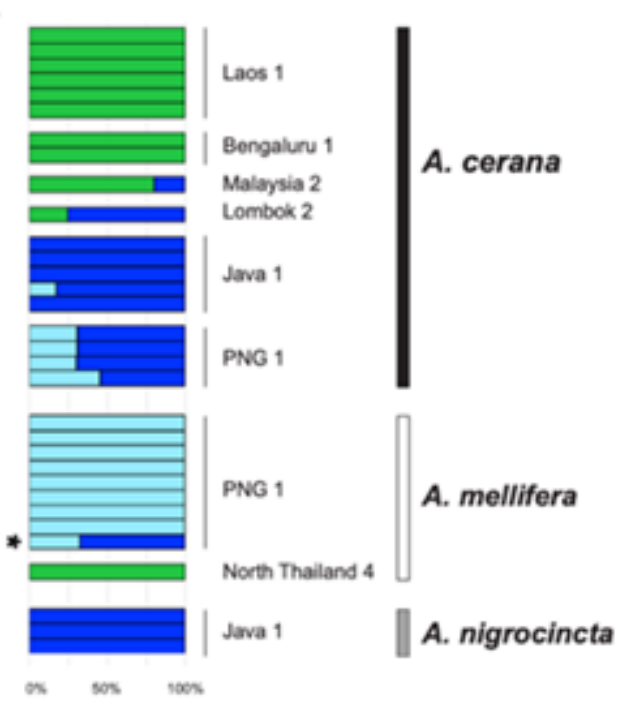

Figure 2

Loss of genetic diversity and rapid genomic differentiation occurred in spite of large founding size and migration between sympatric honey bee hosts. V. destructor populations on its original host A. cerana (black circle) show geographic structure across their native range (A). Envelopes around the PCA points are color-coded according to nuclear genetic ancestry (B). Host-switched populations (white square) are genetically homogenous throughout Asia and are most similar to the populations with the same mitotype (K1) (B). Despite a more recent host switch ( 2008), a similar pattern is observed for V. jacobsoni in South-Asia and Oceania, where the PNG1 lineage underwent a host switch (C). However, other switches appear possible elsewhere in $\mathrm{V}$. jacobsoni's range, such as the specimen collected from A. mellifera in North Thailand (green cluster) (D). In both species, there is evidence of mites drifting between hosts, as indicated by genetic analysis of their gut contents, indicated by asterisks. The overall picture for both species is similar, where reproductive differentiation after host switches is coupled with additional 
opportunities for gene flow via drifting, and potential for additional switches happening elsewhere in the range. Note: The designations employed and the presentation of the material on this map do not imply the expression of any opinion whatsoever on the part of Research Square concerning the legal status of any country, territory, city or area or of its authorities, or concerning the delimitation of its frontiers or boundaries. This map has been provided by the authors.

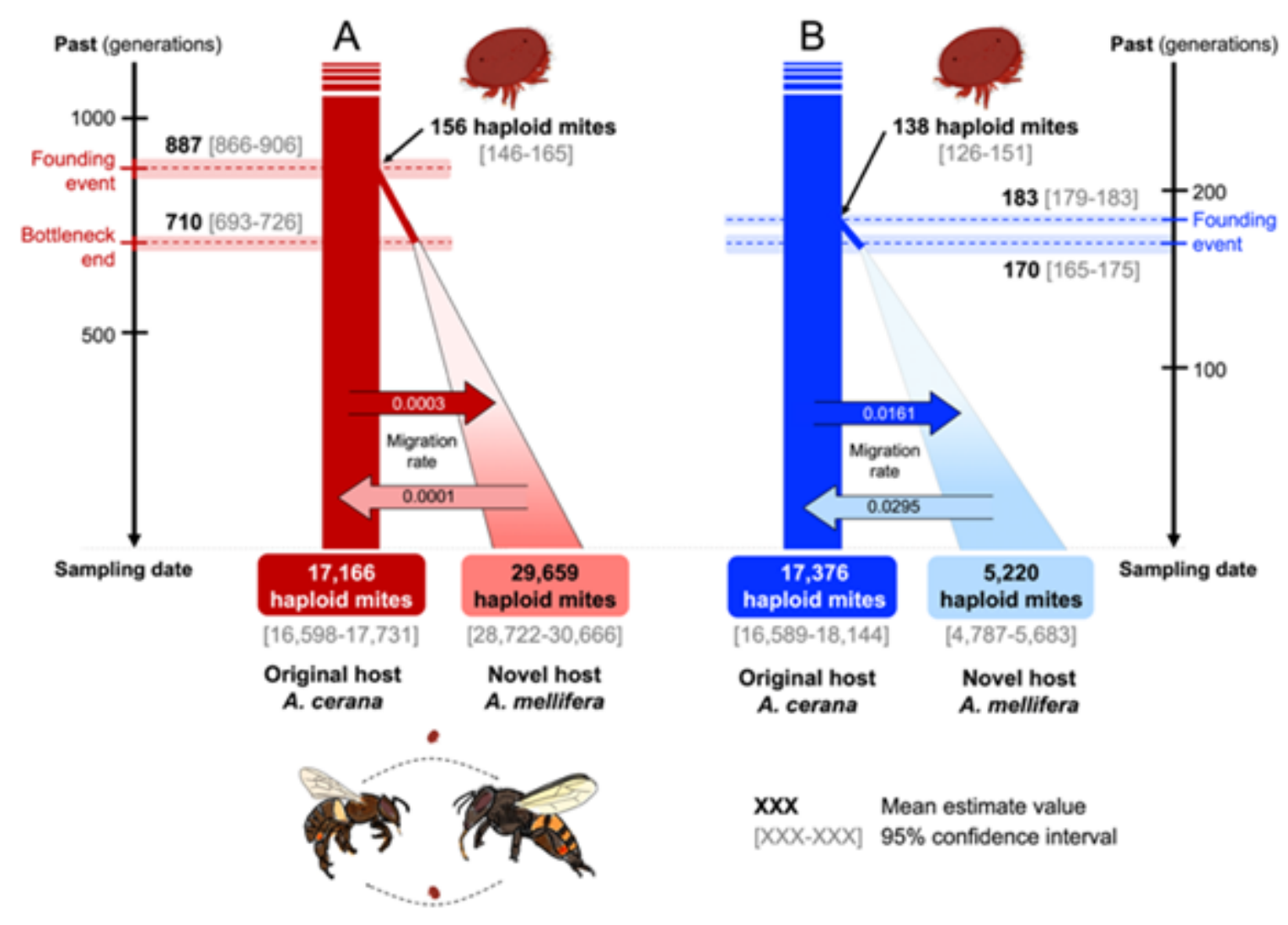

Figure 3

Multiple jumps to A. mellifera and isolation with migration likely allowed V. destructor and V. jacobsoni to successfully switch hosts and persist. Graphical illustration of the inferred scenario with mean parameter estimates in bold and associated $95 \%$ confidence interval in [light gray] with isolation with bidirectional migration (A) for V. destructor and (B) for V. jacobsoni. Estimated population sizes are given in haploid genome size. Migration rates were kept as default values in haploid genome per generation. The coalescent model reconstructed timings of expansion that are consistent with known observations. Yet it suggests that the host switch was not instantaneous and may have involved a period of progressive adaptation before the mites spread beyond their source populations.

\section{Supplementary Files}

This is a list of supplementary files associated with this preprint. Click to download.

- SupplementarytablesDec2020.xIsx 
- SupplementalFigures.docx

Page $30 / 30$ 